\title{
Inventário florístico de samambaias e licófitas de um remanescente de Mata Atlântica no estado do Rio Grande do Sul, Brasil
}

\author{
Checklist of ferns and lycophytes in a remnant \\ of Atlantic Forest in the state of Rio Grande do Sul, Brazil
}

Felipe Gonzatti ${ }^{1,2}$

\begin{abstract}
Resumo
Um inventário de samambaias e licófitas da Reserva Biológica Estadual Mata Paludosa (REBIO Mata Paludosa), Itati, Rio Grande do Sul, Brasil, é apresentado. Durante o período de um ano, as espécies ocorrentes na REBIO foram registradas, com amostras coletadas e incorporadas no herbário HUCS. As espécies foram classificadas quanto ao hábito, grau de ameaça e sua ocorrência nas distintas fitofisionomias do Rio Grande do Sul. No total foram encontradas 79 espécies, sendo uma licófita e 78 samambaias. As famílias de maior riqueza florística foram Polypodiaceae (12), Dryopteridaceae, Pteridaceae e Thelypteridaceae (10 espécies cada) e Aspleniaceae (nove). As plantas terrestres foram as mais representativas (64\%), seguidas pelas epífitas (28\%) e hemiepífitas (8\%). A maioria das espécies tem padrão de distribuição amplo no Rio Grande do Sul (75\%). Encontrou-se populações de cinco espécies localmente ameaçadas de extinção. A REBIO Mata Paludosa representa importantes remanescentes da Mata Atlântica no Estado do Rio Grande do Sul, por apresentar elevada riqueza florística de samambaias e licófitas, espécies de distribuição restrita e ameaçadas de extinção localmente.

Palavras-chave: espécies ameaçadas, flora do Rio Grande do Sul, Mata Atlântica, pteridófitas, Unidades de Conservação.
\end{abstract}

\begin{abstract}
A checklist of ferns and lycophytes in protected area Reserva Biológica Estadual Mata Paludosa (REBIO Mata Paludosa), municipality of Itati, Rio Grande do Sul, Brazil is presented. During one year, a scan survey was made in all vegetation types where the species were collected and incorporated in the HUCS herbarium. The species were categorized by habit, threat level and distribution pattern on Rio Grande do Sul phytophysiognomies. A total of 79 species were recorded, including one lycophyte and 78 ferns. The families Polypodiaceae (12), Dryopteridaceae, Pteridaceae, and Thelypteridaceae (10 each) and Aspleniaceae (nine) were the richest. Terrestrial species were the most representative $(64 \%)$ followed by epiphytes $(28 \%)$, and hemiepyphytes (8\%). Most of the recorded species present are widespread (75\%). Populations of five locally endangered species were found. The REBIO Mata Paludosa represents important remnants of Atlantic Forest in the State of Rio Grande do Sul, because of its high richness of ferns and lycophytes, endangered species and species with locally restrict distribution patterns.
\end{abstract}

Key words: endangered species, Rio Grande do Sul flora, Atlantic Forest, pteridophytes, protected areas.

\section{Introdução}

A biodiversidade brasileira é reconhecida como uma das mais diversas do mundo (Lewinsohn \& Prado 2005; Murray-Smith et al. 2009). Esse reconhecimento é devido à presença de biomas peculiares como a Mata Atlântica, que apresenta mais de 15.700 espécies vegetais, das quais $45 \%$ são endêmicas (Stehmann et al. 2009).

Com referência aos padrões de diversidade e distribuição das samambaias e licófitas, o

\footnotetext{
'Herbário da Universidade de Caxias do Sul, R. Francisco Getúlio Vargas 1130, Bairro Petrópolis, 95070-560, Caxias do Sul, RS, Brasil.

${ }^{2}$ Autor para correspondência: fgonzatti@ucs.br
} 
bioma Mata Atlântica representa um importante complexo de ecossistemas para o crescimento de inúmeras linhagens dos grupos (Salino \& Almeida 2008), devido às características de clima, relevo e heterogeneidade ambiental, que dão origem a ambientes peculiares e considerados ótimos para o estabelecimento das espécies (Tryon \& Tryon 1982; Moran 2008). Atualmente estimase que na Mata Atlântica ocorram cerca de 834 espécies, com aproximadamente 320 endêmicas (38\%) (Prado \& Hirai 2014), o que corrobora este bioma como um dos centros primários de riqueza e endemismos para as espécies na região Neotropical (Tryon 1972).

Apesar de ampla distribuição geográfica de muitas espécies através da dispersão à longa distância por esporos (Tryon 1970; Barrington 1993; Wolf et al. 2001), algumas espécies de samambaias e licófitas apresentam restrições às condições ecológicas, principalmente quanto à temperatura, umidade, estrutura e qualidade da matriz florestal e composição dos substratos, tanto para o desenvolvimento dos esporófitos, como para a germinação de esporos e fixação dos gametófitos (Page 1979). Tais condições fazem com que a distribuição das espécies ao longo dos ecossistemas não seja uniforme, tornando algumas espécies raras, ou com distribuição limitada à habitats muito particulares, e consequentemente passíveis à efeitos extintivos (Mehltreter 2010).

As Unidades de Conservação (UCs) garantem a manutenção e reestabelecimento de ecossistemas naturais sem, ou com baixa interferência humana, protegendo hábitats e nichos ecológicos peculiares (Dudley 2008), bem como seus serviços ecológicos (Fonseca et al. 2010). Atuam também na divulgação da biodiversidade e como espaços de formação. No entanto, apesar do largo histórico de criação e implementação de UCs (Rylands \& Brandon 2005; Mittermeier et al. 2005; Joly et al. 2014), atualmente, somente $1,6 \%$ do bioma Mata Atlântica está legalmente protegido por meio destas (Ribeiro et al. 2009).

No Brasil, tem-se avançado nos últimos anos no conhecimento da taxonomia, ecologia e distribuição das espécies de samambaias e licófitas (Almeida \& Salino 2016). Abordagens de cunho conservacionista foram discutidas por Windisch $(1996,2002)$ apontando um diagnóstico do conhecimento, amostragem e conservação das espécies de plantas vasculares sem sementes. Importantes contribuições vêm sendo incorporadas através da criação de listas florísticas, decretos e resoluções que apontam espécies endêmicas e ameaçadas através da aplicação de critérios estabelecidos pela International Union for Conservation of Nature (IUCN) (Salino 2000; Sylvestre \& Windisch 2003; Aleixo 2006; Santiago 2006; Sylvestre 2007; Salino \& Almeida 2008; Martinelli \& Moraes 2013; Rio Grande do Sul 2014; Gasper \& Salino 2015).

Tais iniciativas tornam-se instrumentos relevantes para o incremento do conhecimento sobre as espécies e podem embasar futuras perspectivas quanto a criação de planos de manejo específicos, no entanto, ainda são necessários esforços no inventário e compilação de dados florísticos quanto a ocorrência e distribuição geográfica das populações das espécies em geral (Almeida \& Salino 2016). No caso específico do Rio Grande do Sul, existem cerca de 80 unidades de conservação sob responsabilidade das esferas Federal, Estadual, Municipal e iniciativa privada, o que perfazem um montante de $7.506,59 \mathrm{~km}^{2}$, ou seja, $2,67 \%$ da área superficial do estado (Backes 2012). Das unidades de conservação presentes no Rio Grande do Sul, muitas ainda carecem de planos de manejo bem estruturados, com inventários detalhados da flora local (Backes 2012).

No que diz respeito samambaias e licófitas, poucos são os estudos florísticos realizados em unidades de conservação no RS, que buscam o incremento do conhecimento das espécies locais e que visam o auxílio no reconhecimento das mesmas pelos gestores e comunidade científica em geral. Dentre estes incluem-se Schmitt et al. (2006) na Floresta Nacional de Canela; Goetz et al. (2012) no Parque Natural Municipal de Ronda; Schmitt \& Goetz (2010) no Parque Municipal Henrique Luiz Roessler, e Becker et al. (2013) no Parque Natural Municipal Tupancy.

O objetivo do presente trabalho é apresentar o inventário florístico das samambaias e licófitas da Reserva Biológica Estadual Mata Paludosa, listando as espécies ocorrentes na área, seu padrão de distribuição nas diferentes regiões fitogeográficas do Rio Grande do Sul, seu hábito e o grau de ameaça conforme a Lista Vermelha das Espécies Ameaçadas de Extinção do RS. Tais resultados visam contribuir no conhecimento da flora de samambaias e licófitas do RS, sua composição florística e a distribuição geográfica das espécies, bem como prover dados para o futuro plano de manejo da unidade. 


\section{Material e Métodos}

Área de estudo

A Reserva Biológica Estadual da Mata Paludosa (REBIO Mata Paludosa) é uma Unidade de Proteção Integral, instituída pelo decreto estadual 38.972 de 23 de outubro de 1998. O propósito da criação desta unidade foi a necessidade de conservar remanescentes da Floresta Atlântica no Rio Grande do Sul, principalmente de fragmentos da Floresta Ombrófila Densa Submontana, localizadas entre a cota de 30 e $400 \mathrm{~m}$ de altitude e da Floresta Atlântica de Terras Baixas situadas entres 5 e $30 \mathrm{~m}$ de altitude (Backes 2012). Um dos ambientes mais importantes da área, é a formação de mata paludosa ou turfosa (de onde deriva o nome da REBIO). Tal formação ocorre nas áreas de depressão do terreno, onde devido à baixa drenagem do solo, compõe uma mata com o solo recoberto pela água permanentemente ou intermitentemente (Waechter 1985).

Anteriormente à implantação da REBIO, as áreas eram utilizadas para cultivos agrícolas, principalmente no desenvolvimento da bananicultura, cana de açúcar, milho, feijão e pecuária. Atualmente, a situação fundiária da REBIO ainda não foi completamente resolvida, sendo que somente 126,48 ha $(46,51 \%$ do total de 271,87 ha) da área total da UC é própria do estado, o restante permanece na posse de proprietários particulares.

Aárea da REBIO está localizada no município de Itati, litoral norte do estado do RS (Fig. 1). A região é uma área de transição entre as formações geomorfológicas da Planície Costeira e Planalto das Araucárias, apresentando amplas áreas de flancos e escarpas rochosas nas faces voltadas para o Planalto (noroeste), até formações arenosas (faces nordeste e leste), da Planície Costeira Quaternária (Poester 2013). A área da REBIO possui um gradiente altitudinal que varia entre 25 e $350 \mathrm{~m}$, partindo da região mais baixa na porção leste, até as mais elevadas na porção oeste.

Os solos que compõem a região são provenientes de duas matrizes geológicas distintas. Nas formações de planície, são encontrados os Argissolos e Gleissolos, que possuem origem

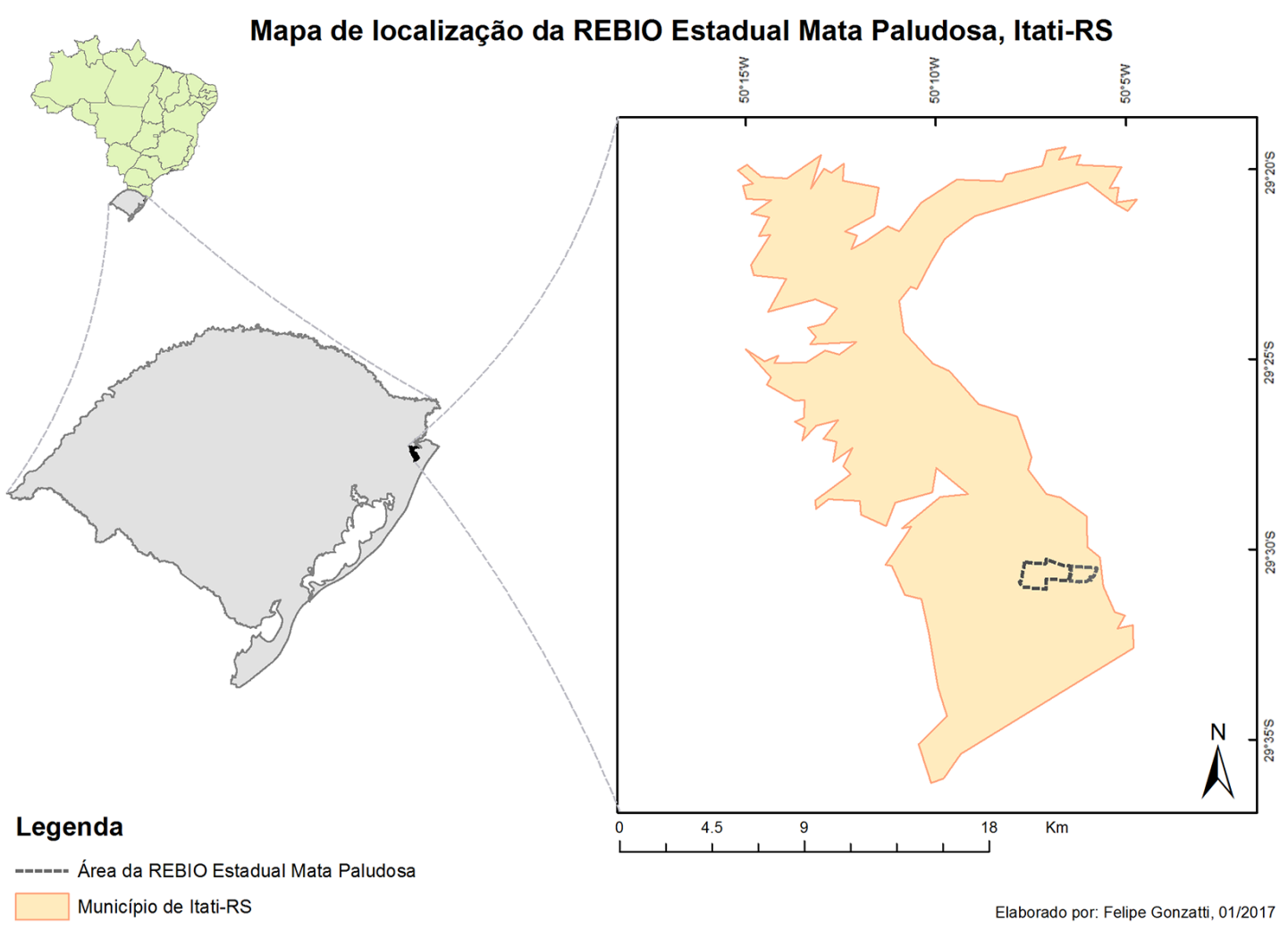

Figura 1 - Mapa de localização da REBIO Estadual Mata Paludosa, e do município de Itati, Rio Grande do Sul, Brasil. Figure 1 - Localization map of State REBIO Mata Paludosa and Itati municipality, of the state of Rio Grande do Sul, Brazil. 
arenosa e deposicional, formados pelos processos mais antigos de transgressão e regressão marinhos quaternários (Tomazelli \& Villwock 1996). Já nas regiões de encosta (cotas entre 50-800 m), são encontrados Chernossolos e Neossolos Litólicos, provenientes da decomposição das rochas basálticas e areníticas das formações Serra Geral e Botucatu respectivamente (EMBRAPA 2006).

O clima da região é classificado como Cfa, segundo a classificação climática Köppen, característico de áreas litorâneas de baixa altitude (abaixo de 500-700 m). Tal clima proporciona precipitações anuais superiores a $1.200 \mathrm{~mm}$ anuais e temperatura média anual de $17,6^{\circ} \mathrm{C}$. A média da temperatura no período do inverno não ultrapassa um mínimo de $-3{ }^{\circ} \mathrm{C}$ e $22{ }^{\circ} \mathrm{C}$ nos meses mais quentes (Moreno 1961; Ferraro 2013).

A vegetação que recobre a área de estudo é composta por distintas fitofisionomias que podem ser encontradas mescladas devido ser uma área de ecótono. Na REBIO Mata Paludosa, podese encontrar desde elementos provenientes das Formações Pioneiras, bem como remanescentes de Floresta Ombrófila Densa de Terras Baixas e Submontana (Backes 2012; Poester \& Brack 2013). Como elementos característicos das Formações Pioneiras podemos encontrar espécies como o maricá [Mimosa bimucronata (DC.) Kuntze], a figueira-de-folha-miúda (Ficus cestrifolia Schott ex Spreng.) e o jerivá [Syagrus romanzoffiana (Cham.) Glassman]. Nos fragmentos de Floresta Ombrófila Densa destacam-se as espécies de peroba [Aspidosperma olivaceum Müll.Arg.], o baguaçu (Talauma ovata A.St.-Hil.) e espécies de cecrópias (Cecropia spp.) formando o dossel da mata. No subbosque encontram-se espécies de arecáceas como o tucum (Bactris setosa Mart.), o palmiteiro (Euterpe edulis Mart.) e as ouricanas (Geonoma schottiana Mart. e G. gamiova Barb.Rodr.).

\section{Amostragem}

As coletas foram realizadas no período de janeiro a dezembro de 2016, com visitas trimestrais. Toda a área da UC foi percorrida, incluindo também áreas lindeiras, como bordos de cultivos e estradas (Fig. 2a-e). As espécies foram coletadas e herborizadas por meio das técnicas usuais conforme descrito por Croft (1999). Todo o material coletado foi incorporado no Herbário da Universidade de Caxias do Sul (HUCS) (acrônimo conforme Thiers, continuamente atualizado). Em campo, anotações acerca das características ecológicas foram realizadas, bem como registros fotográficos. O sistema de classificação adotado seguiu o proposto pelo The Pteridophyte Phylogeny Group (PPG I 2016). A abreviação dos autores das espécies seguiu a Flora do Brasil 2020 (2017).

As categorias de ameaça às espécies foram aplicadas por meio da consulta à Lista Vermelha das Espécies Ameaçadas de Extinção do estado do Rio Grande do Sul (Rio Grande do Sul 2014), a qual utiliza os critérios e categorias utilizadas pela IUCN para avaliação das espécies em escala local. As espécies que não constam na Lista Vermelha foram consideradas como LC (Menos Preocupantes). O hábito preferencial foi considerado por meio das observações de campo e consulta bibliográfica, sendo: espécies terrestres (inclusas aqui também as reofíticas e rupícolas), epifíticas ou hemiepifíticas. Também foi observado o padrão de distribuição das espécies nas distintas regiões fitoecológicas do estado do Rio Grande do Sul, conforme classificação de Veloso (1992), sendo: espécies de distribuição restrita, compreendendo aquelas que ocorrem em somente uma região fitoecológica; espécies de distribuição moderada, as que ocorrem em duas regiões fitoecológicas e, espécies de distribuição ampla, as que ocorrem em três ou mais regiões fitoecológicas. Os dados de ocorrência foram compilados através de consulta bibliográfica, observações de campo e consulta às coleções de herbário.

\section{Resultados e Discussão}

O inventário da REBIO Estadual Mata Paludosa apresenta a ocorrência de 79 espécies em 45 gêneros e 18 famílias (Tab. 1), sendo registrada apenas uma espécie de licófita (Selaginella muscosa, Selaginellaceae, Fig. 3c). As famílias mais representativas foram Polypodiaceae (12), Dryopteridaceae, Pteridaceae, Thelypteridaceae (10 espécies cada) e Aspleniaceae (nove). Anemiaceae, Dicksoniaceae, Didymochlaenaceae, Marattiaceae, Nephrolepidaceae, Osmundaceae e Tectariaceae apresentaram somente uma espécie cada. Os gêneros mais representativos foram Asplenium com nove espécies e Blechnum com quatro, sendo que 30 gêneros foram registrados com somente uma espécie cada (Tab. 1).

A área da REBIO Mata Paludosa representa importantes remanescentes da Floresta Ombrófila Densa para ocorrência da flora de samambaias e licófitas no extremo Sul do Brasil. Foram encontradas espécies características da formação paludosa como Asplenium serra, Campyloneurum 

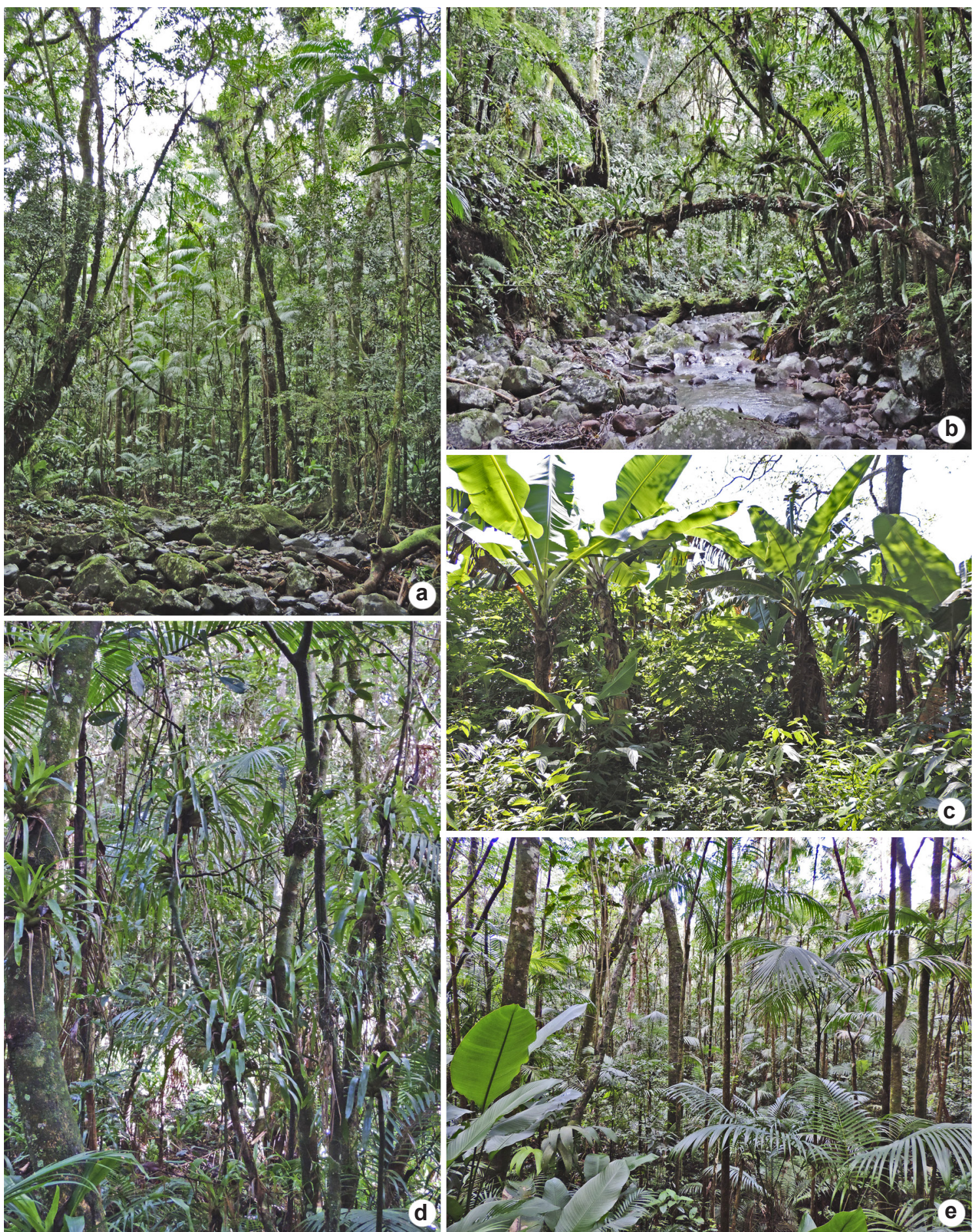

Figura 2 - a-e. Ambientes encontrados na área de estudo - a,b,d,e. formações naturais de Floresta Ombrófila Densa; c. áreas antropizadas com cultivos de banana.

Figure 2 - a-e. Environments found in study area - a,b,d,e. natural formations of Ombrophilous Dense Forest; c. anthropized environments by banana cultivation. 

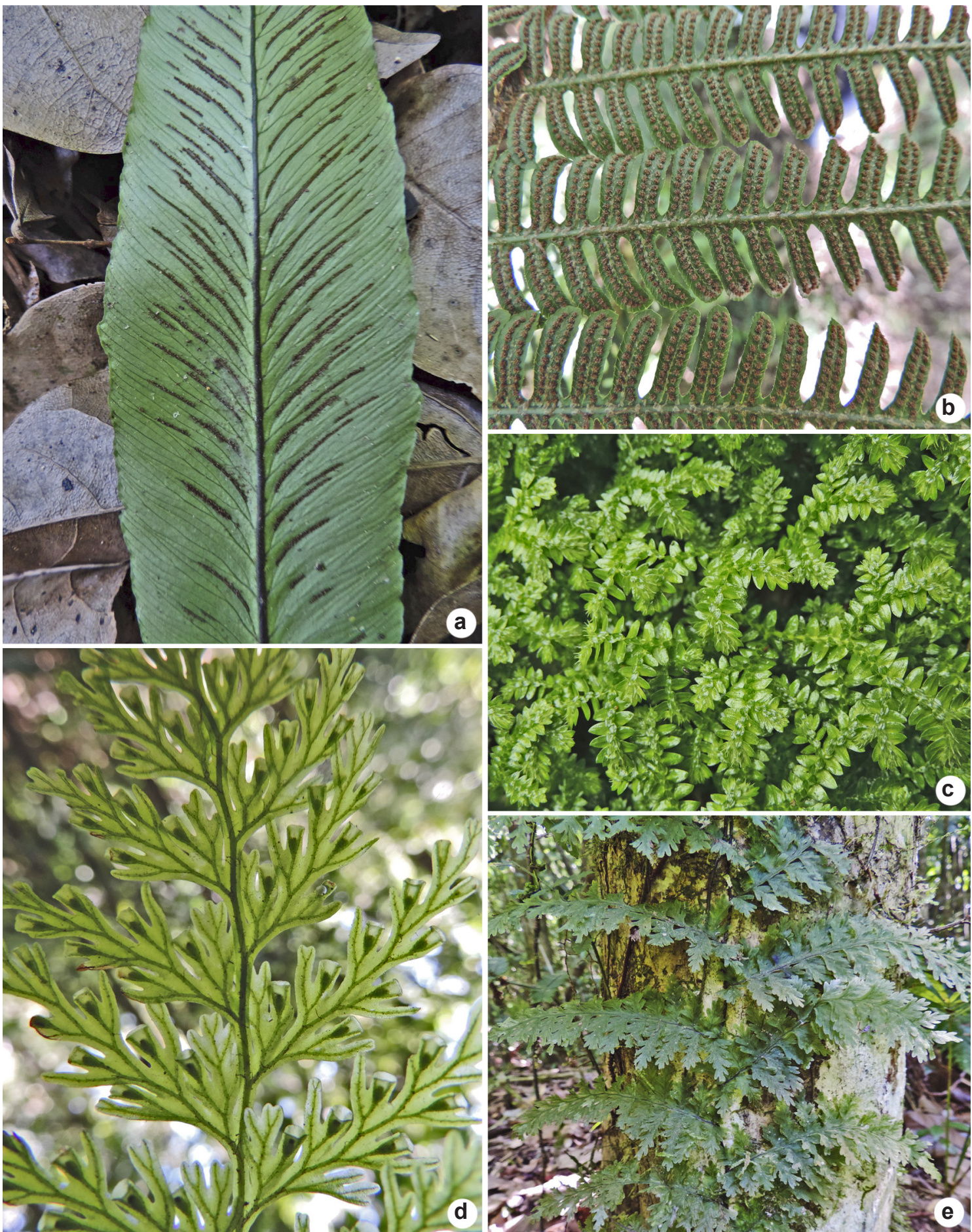

Figura 3 - a-e. Algumas espécies encontradas na área de estudo - a. Diplazium plantaginifolium; b. Ctenitis anniesii; c. Selaginella muscosa; d,e. Vandenboschia rupestris.

Figure 3 - a-e. Some species found in study area - a. Diplazium plantaginifolium; b. Ctenitis anniesii; c. Selaginella muscosa; d,e. Vandenboschia rupestris. 
Tabela 1 - Lista de espécies de samambaias e licófitas encontradas na REBIO Estadual Mata Paludosa-Categoria de ameaça: $\mathrm{VU}=$ vulnerável; $\mathrm{EN}=$ em perigo. Hábito: $\mathrm{T}=$ terrestre; $\mathrm{E}=$ epífita; $\mathrm{H}=$ hemiepífita. Padrão de distribuição das espécies no estado do Rio Grande do Sul: $\mathrm{A}=$ ampla; $\mathrm{M}=$ moderada; $\mathrm{R}=$ restrita.

Table 1 - List of ferns and lycophytes recorded at REBIO Estadual Mata Paludosa - Conservation status: VU = vulnerable; EN = endangered. Habit: $\mathrm{T}$ = terrestrial; $\mathrm{E}=$ epiphyte; $\mathrm{H}=$ hemiepiphyte. Distribution patterns on Rio Grande do Sul state. $\mathrm{A}=$ wide; $\mathrm{M}=$ moderate; $\mathrm{R}=$ restrict.

\begin{tabular}{|c|c|c|c|c|}
\hline Família / Espécie & HUCS & $\begin{array}{l}\text { Categoria } \\
\text { de ameaça }\end{array}$ & Hábito & $\begin{array}{c}\text { Distribuição } \\
\text { no RS }\end{array}$ \\
\hline \multicolumn{5}{|l|}{ Anemiaceae } \\
\hline Anemia phylitidis (L.) Sw. & 44641 & - & $\mathrm{T}$ & A \\
\hline \multicolumn{5}{|l|}{ Aspleniaceae } \\
\hline Asplenium brasiliense Sw. & 44666 & - & $\mathrm{T}$ & A \\
\hline Asplenium gastonis Fée & 44618 & - & $\mathrm{E}$ & A \\
\hline Asplenium inaequilaterale Willd. & 44619 & - & $\mathrm{T}$ & A \\
\hline Asplenium kunzeanum Klotzsch ex Rosenst. & 44605 & - & $\mathrm{T}$ & A \\
\hline Asplenium mucronatum Presl. & 44644 & VU & $\mathrm{E}$ & $\mathrm{R}$ \\
\hline Asplenium oligophyllum Kaulf. & 44647 & - & $\mathrm{E}$ & A \\
\hline Asplenium scandicinum Kaulf. & 44601 & - & $\mathrm{E}$ & A \\
\hline Asplenium serra Langsd. \& Fisch. & 45522 & - & $\mathrm{T}$ & M \\
\hline Asplenium uniseriale Raddi & 44668 & - & $\mathrm{T}$ & M \\
\hline \multicolumn{5}{|l|}{ Athyriaceae } \\
\hline Deparia petersenii (Kunze) M. Kato & 44602 & - & $\mathrm{T}$ & A \\
\hline Diplazium cristatum (Desr.) Alston & 44609 & - & $\mathrm{T}$ & A \\
\hline Diplazium plantaginifolium (L.) Urb. & 44610 & - & $\mathrm{T}$ & $\mathrm{R}$ \\
\hline Diplazium riedelianum (Bong. ex Kuhn) Kuhn ex C. Chr. & 44598 & $\mathrm{EN}$ & $\mathrm{T}$ & $\mathrm{R}$ \\
\hline \multicolumn{5}{|l|}{ Blechnaceae } \\
\hline Blechnum austrobrasilianum de la Sota & 44660 & - & $\mathrm{T}$ & A \\
\hline Blechnum gracile Kaulf. & 44670 & VU & $\mathrm{T}$ & M \\
\hline Blechnum occidentale L. & 44634 & - & $\mathrm{T}$ & A \\
\hline Blechnum polypodioides Raddi & 44661 & - & $\mathrm{T}$ & A \\
\hline Lomaridium acutum (Desv.) Gasper \& V.A.O.Dittrich & 44622 & - & $\mathrm{H}$ & A \\
\hline Neoblechnum brasiliense (Desv.) Gasper \& V.A.O.Dittrich & 44613 & - & $\mathrm{T}$ & A \\
\hline \multicolumn{5}{|l|}{ Cyatheaceae } \\
\hline Alsophila setosa Kaulf. & 44652 & - & $\mathrm{T}$ & A \\
\hline Cyathea atrovirens (Langsd. \& Fisch.) Domin & 44616 & - & $\mathrm{T}$ & A \\
\hline \multicolumn{5}{|l|}{ Dennstaedtiaceae } \\
\hline Dennstaedtia obtusifolia (Willd.) T.Moore & 44635 & - & $\mathrm{T}$ & A \\
\hline Pteridium arachnoideum (Kaulf.) Maxon & 45514 & - & $\mathrm{T}$ & A \\
\hline \multicolumn{5}{|l|}{ Dicksoniaceae } \\
\hline Dicksonia sellowiana Hook. & 45520 & VU & $\mathrm{T}$ & A \\
\hline \multicolumn{5}{|l|}{ Didymochlaenaceae } \\
\hline Didymochlaena truncatula (Sw.) J.Sm. & 44599 & - & $\mathrm{T}$ & A \\
\hline \multicolumn{5}{|l|}{ Dryopteridaceae } \\
\hline Ctenitis anniesii (Rosenst.) Copel. & 45515 & - & $\mathrm{T}$ & $\mathrm{R}$ \\
\hline
\end{tabular}




\begin{tabular}{|c|c|c|c|c|}
\hline Família / Espécie & HUCS & $\begin{array}{l}\text { Categoria } \\
\text { de ameaça }\end{array}$ & Hábito & $\begin{array}{c}\text { Distribuição } \\
\text { no RS }\end{array}$ \\
\hline Ctenitis submarginalis (Langsd. \& Fisch.) Ching & 45516 & - & $\mathrm{T}$ & $\mathrm{A}$ \\
\hline Elaphoglossum glaziovii (Fée) Brade & 45531 & - & $\mathrm{E}$ & M \\
\hline Elaphoglossum luridum (Fée) Christ. & 45532 & - & $\mathrm{E}$ & $\mathrm{R}$ \\
\hline Lastreopsis effusa (Sw.) Tindale & 44643 & - & $\mathrm{T}$ & A \\
\hline Megalastrum connexum (Kaulf.) A.R.Sm. \& R.C.Moran & 44653 & - & $\mathrm{T}$ & A \\
\hline Mickelia scandens (Raddi) R.C. Moran et al. & 44639 & - & $\mathrm{H}$ & $\mathrm{R}$ \\
\hline Olfersia cervina (L.) Kunze & 45521 & - & $\mathrm{H}$ & M \\
\hline Polybotrya cylindrica Kaulf. & 44621 & - & $\mathrm{H}$ & M \\
\hline Rumohra adiantiformis (G.Forst.) Ching. & 44650 & - & $\mathrm{T}$ & A \\
\hline \multicolumn{5}{|l|}{ Hymenophyllaceae } \\
\hline Crepidomanes pyxidiferum (L.) Dubuisson \& Ebihara & 44624 & - & $\mathrm{E}$ & A \\
\hline Didymoglossum reptans (Sw.) C. Presl & 44603 & - & $\mathrm{E}$ & $\mathrm{R}$ \\
\hline Polyphlebium angustatum (Carmich.) Ebihara \& Dubuisson & 44614 & - & $\mathrm{E}$ & A \\
\hline Trichomanes polypodioides Raddi & 44659 & - & $\mathrm{E}$ & M \\
\hline Vandenboschia radicans (Sw.) Copel. & 44604 & - & $\mathrm{H}$ & A \\
\hline Vandenboschia rupestris (Raddi) Ebihara \& K.Iwats. & 44658 & - & $\mathrm{H}$ & $\mathrm{R}$ \\
\hline \multicolumn{5}{|l|}{ Marattiaceae } \\
\hline Danaea moritziana C.Presl & 44638 & - & $\mathrm{T}$ & $\mathrm{R}$ \\
\hline \multicolumn{5}{|l|}{ Nephrolepidaceae } \\
\hline Nephrolepis cordifolia (L.) C.Presl & 45530 & - & $\mathrm{T}$ & A \\
\hline \multicolumn{5}{|l|}{ Osmundaceae } \\
\hline Osmunda regalis $\mathrm{L}$. & - & - & $\mathrm{T}$ & A \\
\hline \multicolumn{5}{|l|}{ Polypodiaceae } \\
\hline Campyloneurum acrocarpon Fée & 45518 & - & $\mathrm{T}$ & M \\
\hline Campyloneurum nitidum (Kaulf.) C.Presl. & 44664 & - & $\mathrm{E}$ & A \\
\hline Campyloneurum rigidum J.Sm. & 45519 & - & $\mathrm{E}$ & $\mathrm{R}$ \\
\hline Microgramma squamulosa (Kaulf.) de la Sota & 44617 & - & $\mathrm{E}$ & A \\
\hline Microgramma vacciniifolia (Langsd. \& Fisch.) Copel. & 44597 & - & $\mathrm{E}$ & A \\
\hline Pecluma pectinatiformis (Lindm.) M.G.Price & 44654 & - & $\mathrm{E}$ & A \\
\hline Pecluma sicca (Lindm.) M.G.Price & 44655 & - & $\mathrm{E}$ & A \\
\hline Pecluma truncorum (Lindm.) M.G.Price & 44633 & VU & $\mathrm{E}$ & A \\
\hline Pleopeltis astrolepis (Liebm.) E.Fourn. & 44669 & - & $\mathrm{E}$ & A \\
\hline Pleopeltis hirsutissima (Raddi) de la Sota & 44628 & - & E & A \\
\hline Pleopeltis pleopeltifolia (Raddi) Alston & 44627 & - & $\mathrm{E}$ & A \\
\hline Serpocaulon catharinae (Langsd. \& Fisch.) A.R.Sm. & 44646 & - & $\mathrm{E}$ & A \\
\hline \multicolumn{5}{|l|}{ Pteridaceae } \\
\hline Adiantum pentadactylon Langsd. \& Fisch. & 44607 & - & $\mathrm{T}$ & $\mathrm{R}$ \\
\hline Adiantum raddianum C.Presl & 44600 & - & $\mathrm{T}$ & A \\
\hline Doryopteris concolor (Langsd. \& Fisch.) J.Sm. & 45525 & - & $\mathrm{T}$ & A \\
\hline Doryopteris nobilis (T.Moore) C.Chr. & 44662 & - & $\mathrm{T}$ & $\mathrm{A}$ \\
\hline Doryopteris pentagona Pic.Serm. & 44642 & - & $\mathrm{T}$ & A \\
\hline Pityrogramma chaerophylla (Desv.) Domin & 45527 & - & $\mathrm{T}$ & A \\
\hline
\end{tabular}




\begin{tabular}{|c|c|c|c|c|}
\hline Família / Espécie & HUCS & $\begin{array}{l}\text { Categoria } \\
\text { de ameaça }\end{array}$ & Hábito & $\begin{array}{c}\text { Distribuiçãa } \\
\text { no RS }\end{array}$ \\
\hline Pteris brasiliensis Raddi & 44636 & - & $\mathrm{T}$ & A \\
\hline Pteris deflexa Link. & 44620 & - & $\mathrm{T}$ & A \\
\hline Pteris denticulata $\mathrm{Sw}$. & 44665 & - & $\mathrm{T}$ & A \\
\hline Vittaria lineata (L.) Sm. & 44648 & - & $\mathrm{E}$ & A \\
\hline \multicolumn{5}{|l|}{ Selaginellaceae } \\
\hline Selaginella muscosa Spring & 44672 & - & $\mathrm{T}$ & A \\
\hline \multicolumn{5}{|l|}{ Tectariaceae } \\
\hline Tectaria pilosa (Fée) R.C.Moran & 44671 & - & $\mathrm{T}$ & $\mathrm{R}$ \\
\hline \multicolumn{5}{|l|}{ Thelypteridaceae } \\
\hline Amauropelta amambayensis (Ponce) Salino \& T.E.Almeida & 44629 & - & $\mathrm{T}$ & A \\
\hline Amauropelta regnelliana (C.Chr.) Salino \& T.E.Almeida & 45529 & - & $\mathrm{T}$ & A \\
\hline Amauropelta retusa (Sw.) Pic.Serm. & 31435 & - & $\mathrm{T}$ & A \\
\hline Amauropelta rivularioides (Fée) Salino \& T.E.Almeida & 44615 & - & $\mathrm{T}$ & A \\
\hline Christella conspersa (Schrad.) Á.Löve \& D.Löve & 44663 & - & $\mathrm{T}$ & A \\
\hline Christella dentata (Forssk.) Brownsey \& Jermy & 44631 & - & $\mathrm{T}$ & A \\
\hline Christella hispidula (Decne.) Holttum & 44630 & - & $\mathrm{T}$ & A \\
\hline Goniopteris riograndensis (Lindm.) Ching & 44612 & - & $\mathrm{T}$ & A \\
\hline Goniopteris scabra (C.Presl) Brade & 44637 & - & $\mathrm{T}$ & A \\
\hline Macrothelypteris torresiana (Gaudich.) Ching & 44640 & - & $\mathrm{T}$ & A \\
\hline
\end{tabular}

acrocarpon, Elaphoglossum luridum, Polybotrya cylindrica e Trichomanes polypodioides, corroborando outros autores (Athayde Filho \& Windisch 2006; Záchia \& Waechter 2011; Gonzatti et al. 2016), bem como representantes das formações de Floresta Ombrófila Densa, como Asplenium oligophyllum, A. mucronatum, Ctenitis anniesii (Fig. 3b), Danaea moritziana, Diplazium riedelianum, Mickelia scandens, Olfersia cervina e Vandenboschia rupestris (Fig. 3d,e) (Gasper \& Salino 2015, Gonzatti et al. 2016).

Em relação aos demais inventários realizados em outras regiões da Floresta Ombrófila Densa do Sul do Brasil, observa-se que a riqueza de espécies presente na REBIO Mata Paludosa é menor. Gasper \& Sevegnani (2010) estudando a área do Parque Nacional do Itajaí encontraram 190 táxons para 57.475 ha de formações de Floresta Ombrófila Densa Submontana, Montana e Altomontana. Dittrich et al. (2005) encontraram 81 espécies de samambaias e licófitas em apenas um hectare de Floresta Ombrófila Densa Montana do Parque Estadual do Pico do Morumbi no estado do Paraná. Labiak \& Prado (1998) estudando somente a sinúsia epifítica da Reserva Volta Velha, em
Itapoá, SC, encontraram 59 espécies, 27 táxons de espécies epífitas a mais do encontrado no presente estudo. A menor riqueza de samambaias e licófitas evidenciada no presente estudo frente a inventários realizados na Floresta Ombrófila Densa dos estados de Santa Catarina e Paraná deve-se também ao efeito da diminuição latitudinal de diversidade (Moran 2008; Gonzatti et al. 2016).

A REBIO Mata Paludosa apresenta 35\% de todas as espécies de samambaias e licófitas ocorrentes na região costeira do RS (Gonzatti et al. 2016) e $21 \%$ da flora destes grupos citadas para o RS [Flora do Brasil 2020 (2017)], tendo o maior número de espécies dentre os inventários em Floresta Ombrófila Densa no estado. Inventários realizados em áreas próximas, ou com similaridade fitofisionômica (Matas de restinga e Floresta Ombrófila Densa), resultaram em números de espécies menos expressivos. Santos \& Windisch (2008), encontraram, nas encostas atlânticas da região de Osório (RS), 53 espécies. Athayde Filho \& Windisch (2006) estudando remanescentes de matas de restinga de Xangri-lá (RS), encontraram 26 espécies em matas paludosas e matas de restinga seca. Nestes mesmos ambientes costeiros, Gonzatti 
et al. (2014a) registraram 65 espécies para o Litoral Médio do RS.

A sobrepujança da riqueza florística da Floresta Ombrófila Densa dentre as formações fitogeográficas ocorrentes no RS foi também evidenciada por Waechter $(1992,1998)$ estudando a distribuição latitudinal de Orchidaceae e de epífitos vasculares. Segundo Sehnem (1977) as florestas Ombrófila Densa e Mista são as mais ricas em espécies de samambaias e licófitas, em contraposição às formações campestres e matas estacionais do Oeste do RS. Este padrão também foi observado por Gasper \& Salino (2015), onde apontam as formações fitogeográficas da Floresta Ombrófila Densa como mais ricas em espécies de samambaias e licófitas frente às formações Ombrófilas Mistas e Estacionais no estado de Santa Catarina, além de maior número de espécies exclusivas presente nesta fitofisionomia.

Quanto o padrão de distribuição nas regiões fitoecológicas do RS, foram encontradas 59 espécies $(75 \%)$ com distribuição ampla, oito $(10 \%)$ com distribuição moderada e 12 (15\%) com distribuição restrita (Fig. 4).

Embora haja uma prevalência de espécies de ampla distribuição geográfica dentre as distintas fitofisionomias ocorrentes no RS (Sehnem 1977; Lorscheitter et al. 1998; 1999; 2001; 2002; 2005; 2009), algumas espécies como Diplazium plataginifloium (Fig. 3a), D. riedelianum (Fig. 5b), Danaea moritziana, Campyloneurum rigidum, Adiantum pentadactylon (Fig. 5d), e outras (Tab.

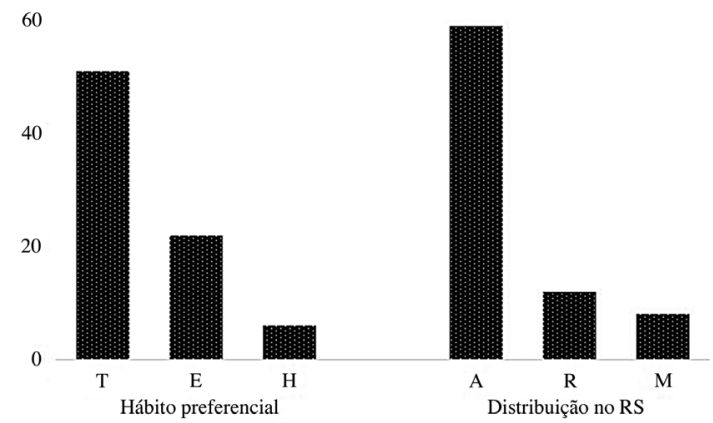

Figura 4 - Hábito preferencial das espécies encontradas na área de estudo $-\mathrm{T}=$ terrestre; $\mathrm{E}=$ epífitas; $\mathrm{H}=$ hemiepífitas. Padrão de distribuição das espécies encontradas na área de estudo $-\mathrm{A}=$ ampla; $\mathrm{M}=$ moderada; $\mathrm{R}=$ restrita.

Figure 4 - Preferential habit of species in the study area $-\mathrm{T}=$ terrestrial; $\mathrm{E}=$ epiphyte; $\mathrm{H}=$ hemiepiphyte. Distribution pattern of species found in study area $-\mathrm{A}=$ wide; $\mathrm{M}=$ moderate; $\mathrm{R}=$ restrict.
1), apresentam no RS distribuição limitada às formações de Floresta Ombrófila Densa. Tais espécies alcançam o RS através do corredor da Mata Atlântica (Porta de Torres) (Rambo 1951), limitando sua distribuição ao extremo norte da região costeira do estado, muitas vezes ocorrendo em populações disjuntas ou localmente reduzidas (Gonzatti et al. 2016). Estas espécies podem ser consideradas raras no RS devido sua baixa representatividade nas coleções de herbário, nos levantamentos florísticos, e por não serem registradas em paralelos mais austrais do RS. Provavelmente requerem condições ecológicas muito restritas, de umidade, sombreamento e temperatura, as quais não se desenvolvem em ambientes divergentes. Tal fator reforça a importância da REBIO Mata Paludosa na manutenção de populações destas espécies no extremo Sul da Mata Atlântica.

As espécies de distribuição moderada compartilham sua distribuição entre a área de estudo (Floresta Ombrófila Densa) com Áreas de Formações Pioneiras, como o caso de Asplenium serra, Polybotrya cylindrica, Campyloneuron acrocarpon que ocorrem amplamente ao longo das restingas paludosas da Planície Costeira. Outras espécies como Elaphoglossum glaziovii e Trichomanes polypodioides ocorrentes também nas Floresta Ombrófila Mista, e Blechnum gracile e Olfersia cervina, registradas também para as Florestas Estacionais do RS. No entanto, outras espécies como Anemia phyllitidis, Asplenium gastonis, Christella dentata (Fig. 5a), Crepidomanes pixydiferum (Fig. 5e), Deparia petersenii, Didymochlaena truncatula, Microgramma squamulosa, M. vacciniifolia (Fig. 6b), Pteris deflexa (Fig. 6c), dentre outras (Tab. 1), são amplamente distribuídas ao longo das diferentes formações fitogeográficas do RS, podendo ser encontradas em variabilidade de ambientes muito diversificados (Nervo et al. 2010; Farias et al. 2014; Gonzatti et al. 2014b).

O hábito terrestre foi mais representativo dentre as espécies da REBIO, compreendendo 51 espécies (64\%), $22(28 \%)$ apresentaram-se como epífitas e seis (8\%) como hemiepífitas (Fig. 6). Embora haja alguns corpos d'água na área da REBIO, não foram identificadas espécies aquáticas.

A condição florestal ombrófila, juntamente com distintos substratos disponíveis (húmus, rochas, solo e capa de raízes) e as variáveis microclimáticas de temperatura e umidade proporcionadas pela orografia acidentada constituem hábitats 

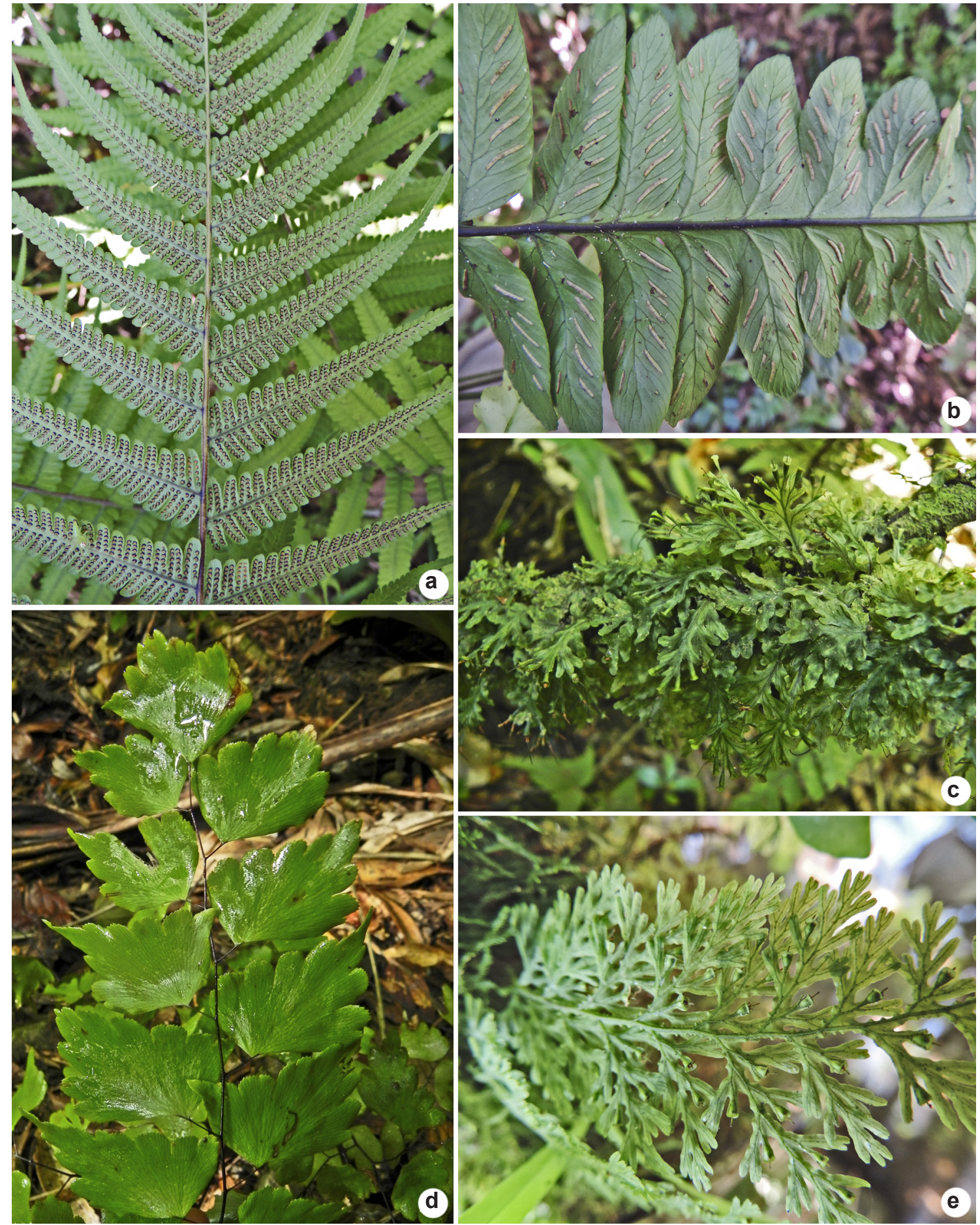

Figura 5 - a-e. Algumas espécies encontradas na área de estudo - a. Christella dentata; b. Diplazium riedelianum; c. Didymoglossum reptans; d. Adiantum pentadactylon; e. Crepidomanes pyxidiferum.

Figure 5 - a-e. Some species found in study area - a. Christella dentata; b. Diplazium riedelianum; c. Didymoglossum reptans; d. Adiantum pentadactylon; e. Crepidomanes pyxidiferum 

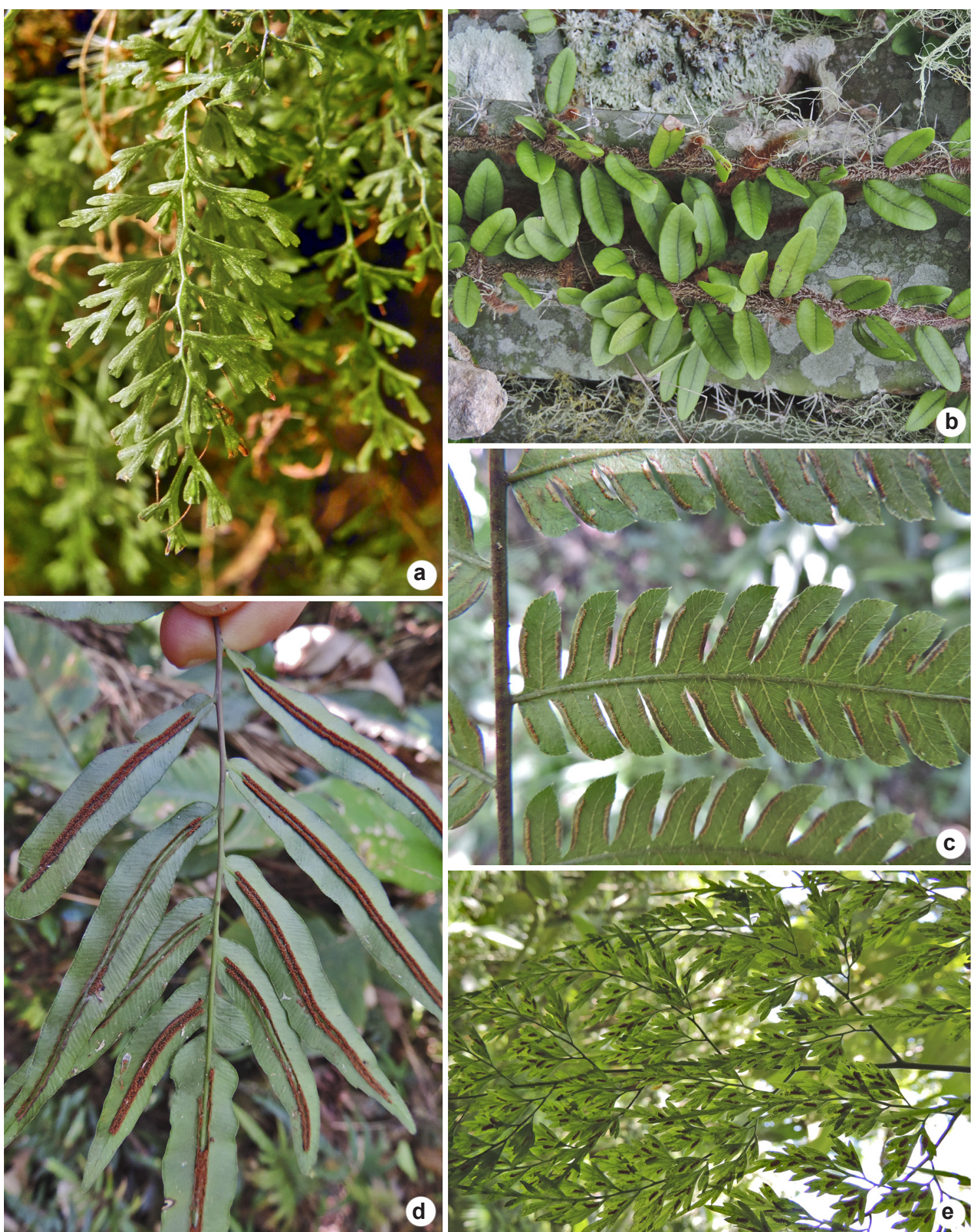

Figura 6 - a-e. Algumas espécies encontradas na área de estudo - a. Polyphlebium angustatum; b. Microgramma vacciniifolia; c. Pteris deflexa; d. Blechnum gracile; e. Asplenium scandicinum.

Figure $\mathbf{6}$ - a-e. Some species found in study area - a. Polyphlebium angustatum; b. Microgramma vacciniifolia; c. Pteris deflexa; d. Blechnum gracile; e. Asplenium scandicinum 
diferenciados e preferenciais para a ocorrência de espécies tropicais de hábito terrestre (Nettesheim et al. 2014), conforme encontrado no presente estudo. Porém, tais condições também favorecem o desenvolvimento de espécies com hábitos de crescimento específicos, como o caso das samambaias hemiepifíticas, como Lomaridium acutum, Mickelia scandens, Olfersia cervina, Vandenboschia rupestris (Fig. 3d,e), V. radicans, ocorrentes com maior frequência neste tipo de fitofisionomia (Orihuela et al. 2013). As espécies epifíticas, como Asplenium scandicinum (Fig. 6e), Didymoglossum reptans (Fig. 5c), Polyphlebium angustatum (Fig. 6a) (Tab. 1), também representam importante contingente florístico, sendo que na área de estudo foram registradas cerca de $64 \%$ de todas as espécies de samambaias e licófitas epifíticas listadas para a região costeira do RS (Machado et al. 2016) e cerca de $9 \%$ de todas as epífitas vasculares ocorrentes na Planície Costeira (Waechter 1992).

Dentre as espécies encontradas, cinco estão listas na Lista Vermelha de espécies ameaçadas de extinção do RS, sendo Asplenium mucronatum, Blechnum gracile (Fig. 6e), Dicksonia sellowiana e Pecluma truncorum na categoria Vulnerável (VU) e Diplazium riedelianum, Em Perigo (EN) (Fig. 5b).

As espécies ameaçadas de extinção encontradas na área estudada representam $6 \%$ de todas as samambaias e licófitas listados no Livro Vermelho da Flora Ameaçada de Extinção do Rio Grande do Sul (73 espécies). Algumas espécies ameaçadas de extinção como Dicksonia sellowiana, que também consta no Livro Vermelho da Flora do Brasil na categoria de EN (Martinelli \& Moraes 2013), tem largo histórico de uso e extrativismo ao longo de sua área de ocupação e necessita de preservação e manejo devido seu importante papel ecológico nos ecossistemas onde ocorre (Windisch 2002). Outras espécies como Diplazium riedelianum e Blechnum gracile estão listadas como espécies ameaçadas devido à particularidade do hábitat o qual ocorrem e por apresentar populações muito reduzidas no RS (Rio Grande do Sul 2014). Já Asplenium mucronatum e Pecluma truncorum, além de apresentarem peculiaridade de hábitat, apresentam estreita relação com os forófitos onde se desenvolvem como epífitas. Na área de estudo, foram encontradas restritamente sobre cáudices de Alsophila setosa.

Durante as expedições de campo, podese evidenciar algumas potenciais ameaças às populações das samambaias e licófitas ocorrentes na área de estudo, como a manutenção de atividades agrícolas, uso de áreas da REBIO para atividades de motocross, supressão parcial de fragmentos lindeiros à Unidade, pisoteio de plântulas e manutenção de trilhas em meio aos remanescentes. Embora a área da REBIO Mata Paludosa seja muito reduzida frente às demais UCs presentes na Planície Costeira, como o Parque Estadual de Itapeva, Parque Nacional da Lagoa do Peixe e Estação Ecológica do Taim (Backes 2012), esta representa uma importante área para a ocorrência de populações de samambaias e licófitas nesta região do RS. Sua maior contribuição se dá principalmente na proteção de espécies da Floresta Ombrófila Densa, as quais apresentam distribuição reduzida no RS e às vezes disjunta às formações florestais do Sudeste do Brasil.

\section{Agradecimentos}

$\mathrm{O}$ autor agradece à M.Sc. Letícia Osório da Rosa, a Olaf Link e a Bárbara Giuriatti, a companhia e auxílio nas amostragens de campo. Também agradece à gestora da REBIO Mata Paludosa, M.Sc. Fernanda Schmitt, o auxílio nas atividades de campo e informações acerca da UC. Ao Dr. Paulo Günter Windisch, e aos revisores, os comentários e sugestões do texto. À Universidade de Caxias do Sul, e à equipe do Herbário HUCS, o apoio e infraestrutura prestada.

\section{Referências}

Aleixo A (2006) Oficina de trabalho "Discussão e elaboração da lista de espécies ameaçadas de extinção do estado do Pará". Museu Paraense Emílio Goeldi, Belém. 40p.

Almeida T \& Salino A (2016) State of the art and perspectives on neotropical fern and lycophyte systematics. Journal of Systematics and Evolution 54: 679-690

Athayde Filho FP \& Windisch PG (2006) Florística e aspectos ecológicos das pteridófitas em uma floresta de Restinga no estado do Rio Grande do Sul, Brasil. Iheringia, Série Botânica 61: 63-71.

Backes A (2012) Áreas protegidas no estado do Rio Grande do Sul: o esforço para conservação. Pesquisas, Botânica 63: 225-355.

Barrington DS (1993) Ecological and historical factors in fern biogeography. Journal of Biogeography 20: 275-280.

Becker DFP, Cunha S, Goetz MNB, Kieling-Rubio MA \& Schmitt JL (2013) Florística de samambaias e licófitas em fragmento florestal da bacia hidrográfica do Rio dos Sinos, Caraá, RS, Brasil. Pesquisas, Botânica, 64: 273-278. 
Croft J (1999) A guide to collecting herbarium specimens of ferns and their allies. Disponível em $<\mathrm{http}$ ://www. anbg.gov.au/fern/collecting.html $>$. Acesso em 15 janeiro 2017.

Dittrich VAO, Waechter JL \& Salino A (2005) Species richness of pteridophytes in a montane Atlantic rain forest plot of Southern Brazil. Acta Botanica Brasilica 19: 519-525.

Dudley N (2008) Guidelines for applying protected area management categories. IUCN, Gland. 86p.

EMBRAPA (2006) Sistema brasileiro de classificação de solos. Empresa Brasileira de Pesquisa Agropecuária. $2^{a}$ ed. EMBRAPA, Rio de Janeiro. 306p.

Farias APS, Leal C, Garlet KTMB \& Essi L (2014) Pteridoflora da Universidade Federal de Santa Maria (UFSM), campus Palmeira das Missões, RS, Brasil. Acta Biológica Catarinense 1: 15-21.

Ferraro LMW (2013) Clima. In: Castro D \& Mello RSP (orgs.). Atlas ambiental da bacia do Rio Tramandaí. Via Sapiens, Porto Alegre. 180p.

Flora do Brasil 2020 (2017) Jardim Botânico do Rio de Janeiro. Disponível em $<$ http://floradobrasil.jbrj. gov.br/reflora/floradobrasil/FB128483>. Acesso em 10 janeiro 2017.

Fonseca M, Lamas I \& Kasecker TO (2010) Papel das unidades de conservação. Scientific American Brasil 39: $18-23$.

Gasper AL \& Salino A (2015) Samambaias e licófitas de Santa Catarina: composição, riqueza e espécies ameaçadas. Iheringia, Série Botânica 70: 321-342.

Gasper AL \& Sevegnani L (2010) Lycophyta e samambaias do Parque Nacional da Serra do Itajaí, Vale do Itajaí, SC, Brasil. Hoehnea 37: 755-767.

Goetz MNB, Fraga LL \& Schmitt JL (2012) Florística e aspectos ecológicos de samambaias e licófitas em um parque urbano do Rio Grande do Sul, Brasil. Pesquisas, Botânica 63: 165-176.

Gonzatti F, Valduga E, Wasum R \& Scur L (2014a) Florística e aspectos ecológicos de licófitas e samambaias do litoral médio do Rio Grande do Sul, Brasil. Revista Brasileira de Biociências 12: 215-225.

Gonzatti F, Valduga E, Wasum R \& Scur L (2014b) Florística e aspectos ecológicos de samambaias e licófitas em remanescentes de matas estacionais deciduais da Serra Gaúcha, Rio Grande do Sul, Brasil. Revista Brasileira de Biociências 12: 90-97.

Gonzatti F, Machado L \& Windisch P (2016) Distribution patterns of ferns and lycophytes in the Coastal Region of the state of Rio Grande do Sul, Brazil. Acta Botanica Brasilica 30: 239-253.

Joly CA, Metzger JP \& Tabarelli M (2014) Experiences from the Brasilian Atlantic Forest: ecological findings and conservation initiatives. New Phytologist 204: 459-473.

Labiak PH \& Prado J (1998) Pteridófitas epífitas da Reserva Volta Velha, Itapoá, Santa Catarina, Brasil. Boletim do Instituto de Botânica 11: 1-79.
Lewinsohn TM \& Prado PI (2005) How many species are there in Brazil? Conservation Biology 19: 619-624.

Lorscheitter ML, Ashraf AR, Bueno RM \& Mosbrugger V (1998) Pteridophyte spores of Rio Grande do Sul flora, Brazil. Part I. Palaeontographica Abteilung B: Palaöphytologie 246: 1-113.

Lorscheitter ML, AshrafAR, Windisch PG \& Mosbrugger V (1999) Pteridophyte spores of Rio Grande do Sul flora, Brazil. Part II. Palaeontographica Abteilung B: Palaöphytologie 251: 71-235.

Lorscheitter ML, AshrafAR, Windisch PG \& Mosbrugger V (2001) Pteridophyte spores of Rio Grande do Sul flora, Bazil. Part III. Palaeontographica Abteilung B: Palaöphytologie 260: 1-165.

Lorscheitter ML, AshrafAR, Windisch PG \& Moseburger V (2002) Pteridophyte spores of Rio Grande do Sul flora, Brazil. Part IV. Palaeontographica Abteilung B: Palaöphytologie 263: 1-159.

Lorscheitter ML, AshrafAR, Windisch PG \& Moseburger V (2005) Pteridophyte spores of Rio Grande do Sul flora, Brazil. Part V. Palaeontographica Abteilung B: Palaöphytologie 270: 1-180.

Lorscheitter ML, AshrafAR, Windisch PG \& Mosbrugger V (2009) Pteridophyte spores of Rio Grande do Sul flora, Brazil. Part VI. Palaeontographica Abteilung B: Palaöphytologie 281: 1-96.

Machado L, Gonzatti F \& Windisch PG (2016) Epiphytic ferns in swamp forest remnants of the coastal plain of southern Brazil: latitudinal effects on the plant community. Acta Botanica Brasilica 30: 644-657.

Martinelli G \& Moraes MA (2013) Livro vermelho da flora do Brasil. Instituto de Pesquisas Jardim Botânico do Rio de Janeiro, Rio de Janeiro. 1002p.

Mehltreter K (2010) Ferns conservation. In: Mehltreter K, Walker LR \& Sharpe JM (eds.). Fern ecology. Cambridge University Press, New York. Pp. 1-18.

Mittermeier R, Fonseca GAB \& Rylands AB (2005) A brief history of biodiversity conservation in Brazil. Conservation Biology 19: 601-607.

Moran RC (2008) Diversity, biogeography and floristic. In: Ranker TA \& Haufler CH (eds.). Biology and evolution of ferns and lycophytes. Cambridge University Press, Cambridge. Pp. 367-394.

Moreno JA (1961) Clima do Rio Grande do Sul. Secretaria da Agricultura, Porto Alegre. 42p.

Murray-Smith C, Neil AB, Oliveira-Filho AT, Bachman S, Moat J, Lughadha EMN \& Lucas E (2009) Plant diversity hotspots in the atlantic coastal forests of Brazil. Conservation Biology 23: 151-163

Nervo MH, Windisch PG \& Lorscheitter ML (2010) Representatividade da base amostral da pteridoflora do estado do Rio Grande do Sul (Brasil) e novos registros de distribuição. Pesquisas, Botânica 61: 245-258.

Nettesheim FC, Damasceno E \& Sylvestre L (2014) Different slopes of a mountain can determine the structure of ferns and lycophytes communities in 
a tropical forest of Brazil. Anais da Academia Brasileira de Ciências 86: 199-210.

Orihuela RLL, Molz M \& Waechter JL (2013) Links between environmental factors and hemiepiphytes along a slope of subtropical Atlantic forest. Nordic Journal of Botany 31: 1-11.

Page CN (1979) Experimental aspects of fern Ecology. In: Dyer AF (ed.). The experimental biology of ferns. Academic Press, London. Pp. 551-589.

Poester OC (2013) Relevo. In: Castro D \& Mello RSP (orgs.). Atlas ambiental da bacia do Rio Tramandaí. Via Sapiens, Porto Alegre. 180p.

Poester OC \& Brack P (2013) Bioma e região fitoecológica. In: Castro D \& Mello RSP (orgs.). Atlas ambiental da bacia do Rio Tramandaí. Via Sapiens, Porto Alegre. 180p.

PPG I (2016) A community-derived classification for extant lycophytes and ferns. Journal of Systematic and Evolution 54: 563-603.

Prado J \& Hirai RY (2014) Biogeography of the Brazilian Atlantic forest: evidence from phylogenetic data sets and perspectives for fern and lycophytes studies. Fern Gazette 19: 241-257.

Rambo B (1951) A imigração da selva higrófila no Rio Grande do Sul. Anais Botânicos do Herbário Barbosa Rodrigues 3: 55-91.

Ribeiro MC, Metzger JP, Martensen AC, Ponzoni FJ \& Hirota MM (2009) Brazilian Atlantic forest: how much is left and how is the remaining forest distributed? Implications for conservation. Biological Conservation 142: 1141-1153.

Rio Grande do Sul (2014) Decreto 52.109 de 19 de dezembro de 2014. Declara as espécies da flora nativa ameaçadas de extinção. Diário Oficial da União, Porto Alegre. Pp. 2-11.

Rylands AB \& Brandon K (2005) Brazilian protected areas. Conservation Biology 19: 612-618.

Salino A (2000) Pteridófitas. In: Mendonça MP \& Lins L (orgs.). Lista vermelha das espécies ameaçadas de extinção da flora de Minas Gerais. Fundação Biodiversitas, Belo Horizonte. 157p.

Salino A \& Almeida TE (2008) Diversidade e conservação das pteridófitas na Cadeia do Espinhaço, Brasil. Megadiversidade 4: 78-98.

Santiago ACPS (2006) Pteridófitas da Floresta Atlântica ao norte do Rio São Francisco: florística, biogeografia e conservação. Tese de Doutorado. Universidade Federal de Pernambuco, Recife. $146 \mathrm{p}$.

Santos ACC \& Windisch PG (2008) Análise da pteridoflora da Área de Proteção Ambiental do Morro da Borússia, Osório, RS. Pesquisas, Botânica 59: 237-252.

Schmitt JL, Fleck R, Burmeister EL \& Kieling-Rubio MA (2006) Diversidade e formas biológicas de pteridófitas da Floresta Nacional de Canela, Rio Grande do Sul: contribuições para o plano de manejo. Pesquisas, Botânica 57: 275-288.
Schmitt JL \& Goetz MNB (2010) Species richness of fern and lycophyte in an urban park in the Rio dos Sinos basin, Southern Brazil. Brazilian Journal of Biology 70: 1161-1167.

Sehnem A (1977) As filicíneas do sul do Brasil, sua distribuição geográfica, sua ecologia e suas rotas de migração. Pesquisas, Botânica 31: 1-108.

Stehmann JR, Forzza RC, Salino A, Sobral M, Costa DP \& Kamino LHY (2009) Diversidade taxonômica na Floresta Atlântica. In: Stehmann JR, Forzza RC, Salino A, Sobral M, Costa DP \& Kamino LHY (orgs.). Plantas da Floresta Atlântica. Instituto de Pesquisas Jardim Botânico do Rio de Janeiro, Rio de Janeiro. Pp. 3-12.

Sylvestre LS (2007) As pteridófitas ameaçadas de extinção no estado do Espírito Santo. In: Simonelli M \& Fraga CN (orgs.). Espécies da flora ameaçadas de extinção no estado do Espírito Santo. Ipema, Vitória. Pp. 89-96.

Sylvestre LS \& Windisch PG (2003) Diversity and distribution patterns of Aspleniaceae in Brazil. In: Chandra S \& Srivastava M (eds.). Pteridology in the New Millennium. Kluwer Academic Publishers, Dordrecht. Pp. 107-120.

Thiers B [continuamente atualizado] Index Herbariorum: a global directory of public herbaria and associated staff. New York Botanical Garden's Virtual Herbarium. Disponível em $<$ http:// sweetgum.nybg.org/science/ih/>. Acesso em 6 janeiro 2017.

Tomazelli LJ \& Villwock JA (1996) Quaternary geological evolution of Rio Grande do Sul coastal plain, Southern Brazil. Anais da Academia Brasileira de Ciências 68: 373-382.

Tryon RM (1970) Development and evolution of fern floras of oceanic islands. Biotropica 2: 76-84.

Tryon RM (1972) Endemic areas and geographic speciation in Tropical American Ferns. Biotropica 4: 121-131.

Tryon RM \& Tryon AF (1982) Ferns and allied plants with special reference to tropical America. Springer, New York. 857p.

Veloso HP (1992) Manual técnico da vegetação brasileira. Manual técnico em geociências. IBGEDERMA, Rio de Janeiro. 92p.

Waechter JL (1985) Aspectos ecológicos da vegetação de restinga no Rio Grande do Sul, Brasil. Comunicação do Museu de Ciências, PUCRS. Série Botânica 33: 49-68.

Waechter JL (1992) O epifitismo vascular na Planície Costeira do Rio Grande do Sul. Tese de Doutorado. Universidade de São Carlos, São Carlos. 163p.

Waechter JL (1998) Epiphytic orchids in eastern subtropical South America. Proceedings of the $15^{\text {th }}$ World Orchid Conference, Rio de Janeiro, Brasil. Naturalia Publications, Turriers. Pp. 332-341.

Windisch PG (1996) Towards assaying biodiversity in Brazilian pteridophytes. In: Bicudo CEM \& 
Menezes NA (eds.). Biodiversity in Brazil: a first approach. CNPq, São Paulo. Pp. 109-117.

Windisch PG (2002) Fern conservation in Brazil. Fern Gazette 16: 295-300.

Wolf PG, Schneider H \& Ranker TA (2001) Geographic distributions of homosporous ferns: does dispersal obscure evidence of vicariance? Journal of Biogeography 28: 263-270.

Záchia RA \& Waechter JL (2011) Diferenciação espacial de comunidades herbáceo-arbustivas em florestas costeiras do Parque Nacional da Lagoa do Peixe, Rio Grande do Sul. Pesquisas, Botânica 62: 211-238. 